\title{
Sulforaphane inhibits angiogenesis through activation of FOXO transcription factors
}

\author{
RACHEL DAVIS $^{1}$, KARAN P. SINGH $^{2}$, RAZELLE KURZROCK $^{3}$ and SHARMILA SHANKAR ${ }^{1}$ \\ ${ }^{1}$ Department of Biochemistry, Center for Biomedical Research, The University of Texas Health Science Center at Tyler, \\ Tyler, TX 75708-3154; ${ }^{2}$ Department of Biostatistics, University of North Texas Health Science Center \\ at Fort Worth, Fort Worth, TX 76107-2699; ${ }^{3}$ Department of Investigational Cancer Therapeutics, \\ The University of Texas M.D. Anderson Cancer Center, Houston, TX 77030, USA
}

Received May 18, 2009; Accepted June 29, 2009

DOI: $10.3892 /$ or_00000589

\begin{abstract}
Recent studies have suggested that sulforaphane, a compound found largely in cruciferous vegetables, could inhibit tumor growth through regulation of angiogenesis. However, the molecular mechanism by which it inhibits angiogenesis has not been reported. In this study, we examined the molecular mechanisms by which sulforaphane (SNF) inhibits angiogenesis through regulation of FOXO transcription factor in human umbilical vein endothelial cells (HUVECs). Inhibition of MEK/ERK and PI3K/AKT pathways synergistically inhibited cell migration and capillary tube formation by HUVECs and further enhanced the antiangiogenic effects of sulforaphane. Inhibitors of MEK and AKT kinases synergistically enhanced nuclear translocation of FOXO3a. Inhibition of the MEK/ERK and PI3K/AKT pathways synergistically induced FOXO transcriptional activity and inhibited cell migration and capillary tube formation; these events were further enhanced in the presence of sulforaphane. Phosphorylation deficient mutants of FOXO enhanced antiangiogenic effects of sulforaphane by activating the FOXO transcription factor. In conclusion, activation of FOXO transcription factor by sulforaphane could be an important physiological process to inhibit angiogenesis which may ultimately control tumor growth. These novel antiangiogenic activities of sulforaphane are likely to contribute to its cancer chemopreventive and therapeutic potential.
\end{abstract}

\section{Introduction}

Epidemiological studies have shown that people who eat cruciferous vegetables have reduced incidence of cancer. Isothiocyanates (ITCs) are sulfur-containing compounds that are broadly distributed among cruciferous vegetables such as cabbages and broccoli (1-3). The consumption of ITCs is

Correspondence to: Dr Sharmila Shankar, Department of Biochemistry, The University of Texas Health Science Center at Tyler, 11937 US Highway 271, Tyler, TX 75708-3154, USA

E-mail: sharmila.shankar@uthct.edu

Key words: sulforaphane, HUVEC, angiogenesis, FOXO expected to rise due to the use of dietary supplements and public health initiatives promoting the consumption of more fruits and vegetables. SFN possesses antioxidant, anti-proliferative, chemopreventive and anti-carcinogenic properties (1-3). SFN inhibits growth of prostate and breast cancer in vivo (4-9), and also prevents chemically induced breast $(10,11)$, stomach (12) and colon (13) cancers in rats. SFN inhibits osteoclastogenesis by inhibiting NFKB (14). It is rapidly absorbed and shows an absolute bioavailability of $82 \%$ (15). SFN induces a phase 2 enzyme, thereby neutralizing carcinogens before they can damage DNA $(16,17)$. SFN is extensively metabolized and can therefore compete with other substrates of phases I, II, III enzymes and transporters. It inhibits benzo[a]pyrene-DNA and 1,6-dinitropyrene-DNA adducts formation. However, the molecular mechanisms by which sulforaphane inhibits angiogenesis are not well understood.

FOXO subfamily of forkhead transcription factors include FOXO1a/FKHR, FOXO3a/FKHRL1, and FOXO4/AFX (18). The phosphoinositides 3-kinase (PI3K) pathway, via activation of its downstream kinase AKT, phosphorylates each of the FOXO proteins (19). Inhibition of the PI3K pathway leads to dephosphorylation and nuclear translocation of active FKHRL1, FKHR, and AFX; which induce cells cycle arrest and apoptosis and inhibit angiogenesis (20). FOXO transcription factors control several genes related to cell cycle and apoptosis (21-25). Consequently, activation of the PI3K pathway serves to repress FOXO-mediated growth arrest and apoptosis. SFN has been shown to downregulate $\mathrm{PI} 3 \mathrm{~K} / \mathrm{AKT}(26,27)$ pathways in vitro. Furthermore, we have recently demonstrated that SFN can inhibit the phosphorylation (activation) of AKT in human prostate cancer cells orthotopically implanted in nude mice (9). However, its effects on the activation of FOXO transcription factors and subsequent inhibition on angiogenesis have not been examined.

In addition to $\mathrm{PI} 3 \mathrm{~K} / \mathrm{AKT}$ pathway, MAP kinases also regulate the phosphorylation of FOXO transcription factors and angiogenesis $(28,29)$. MAPKs may regulate the phosphorylation of FOXO1 (FKHR) which contains 15 consensus phosphorylation sites. In vitro kinase assay showed that FOXO1 was phosphorylated by ERK and p38 but not by JNK (28). In NIH3T3 cells, epidermal growth factor- or aniso- 
mycin-induced phosphorylation of FOXO1 was significantly inhibited by an MEK1 inhibitor (PD98059), or a p38 inhibitor (SB203580). Phosphopeptide mapping using mutation of phosphorylation sites for MAPK revealed that the nine serine residues in FOXO1 are specifically phosphorylated by ERK and that five of the nine residues are phosphorylated by p38 in vivo. In support of this concept, we have recently demonstrated that inhibitors of AKT and MEK1 synergistically induced FOXO transcriptional activity and inhibited in vitro angiogenesis (30). We have also demonstrated that SFN can inhibit the phosphorylation of ERK and FOXO proteins in human prostate tumors orthotopically implanted in nude mice (9). Thus, these studies clearly demonstrate phosphorylationdependent regulation of FOXO by AKT and MAP kinases.

The purpose of this study was to examine whether the inhibition of MEK/ERK and PI3K/AKT pathways enhanced the antiangiogenic effects of sulforaphane through activation of FOXO transcription factors. We have demonstrated that inhibition of MEK/ERK and PI3K/AKT pathways acted synergistically to induce FOXO transcription activity, capillary tube formation and migration. Furthermore, antiangiogenic effects of sulforaphane were regulated through activation of FOXO transcription factors. Thus, sulforaphane may regulate tumor angiogenesis through activation of FOXO transcription factors.

\section{Materials and methods}

Reagents. Sulforaphane was purchased from LKT Laboratories, Inc. (St. Paul, MN). MEK inhibitor (PD98059), and AKT inhibitor-IV were purchased from EMD Biosciences (San Diego, CA). Dual Luciferase Reporter Assay kit was purchased from Promega Corporation (Madison, WI).

Cell culture and cell proliferation assay. Human umbilical vein endothelial cells (HUVECs) were purchased from Clonetics (Walkersville, MD) and maintained in endothelial cell growth factor medium-2 (EGM2 MV SingleQuots, Clonetics) supplemented with 5\% FBS. For cell proliferation assay, HUVECs were seeded in 24-well plates and treated with sulforaphane. Viable cells were counted under a microscope after staining cells with trypan blue dye.

In vitro cell migration assay. Cell migration assay was performed as we described earlier (31). In brief, migration of HUVEC cells was assessed using Transwell Boyden chamber (Corning, Acton, MA) containing a polycarbonated filter with a pore size of $8-\mu \mathrm{M}$. HUVECs $\left(4 \times 10^{4}\right.$ cells in $\left.0.2 \mathrm{ml}\right)$ cells in complete medium was mixed with desired concentration of sulforaphane or DMSO (control), and the cell suspension was added to the upper chamber. The lower chamber contained $0.6 \mathrm{ml}$ of complete medium with the same concentration of sulforaphane or DMSO. Migration through the membrane was determined after $24 \mathrm{~h}$ of incubation at $37^{\circ} \mathrm{C}$. Cells remaining on the topside of the transwell membrane were removed using a cotton swab. The membrane was washed with ice-cold PBS. Cells that had migrated to the underside were fixed with $90 \%$ methanol and stained with Giemsa. Cell migration was quantified by counting the number of cells per field in five random fields.
Capillary tube formation assay. Cell migration assay was performed as described by us earlier (31). In brief, matrigel $(500 \mu 1)$ was added to wells of a 24 -well culture plate and allowed to polymerize for $1 \mathrm{~h}$ at $37^{\circ} \mathrm{C}$. To examine the effects of sulforaphane on in vitro angiogenesis, subconfluent HUVECs were resuspended in complete medium and added to Matrigel containing wells (10x10 4 cells/well), and exposed to sulforaphane or DMSO (control). The plates were incubated at $37^{\circ} \mathrm{C}$ in a humidified atmosphere of $95 \%$ air and $5 \% \mathrm{CO}_{2}$. Capillary tube formation was assessed after $24 \mathrm{~h}$ by counting the total number of capillary-like tubular structures from three randomly chosen fields using an inverted microscope.

Luciferase assay. HUVEC cells were transfected with empty vector, FOXO1-TM, or FOXO3a-TM along with reporter plasmids, p6xDBE-luc and pRL-TK. The FOXO expression vectors (wild-type and phosphorylation deficient mutants) and FOXO-luciferase constructs have been described elsewhere $(30,32,33)$. After $24 \mathrm{~h}$, transfection medium was replaced with culture medium and cells were treated with sulforaphane (10-20 $\mu \mathrm{M})$. After incubation of $24 \mathrm{~h}$, the relative luciferase activity, i.e. firefly enzyme activity divided by that of the Renilla enzyme, was determined using Dual Luciferase Reporter Assay System (Promega) according to the manufacturer's protocol.

Immunofluorescence analysis. For visualization of FOXO3a, HUVEC cells were transiently transfected with plasmids encoding GFP- FOXO3a and grown in multiple well chamber slides. After transfection, cells were pretreated with AKT inhibitor IV $(1 \mu \mathrm{M})$ and/or MEK1/2 inhibitor PD98059 $(10 \mu \mathrm{M})$ for $2 \mathrm{~h}$, followed by treatment with or without sulforaphane $(20 \mu \mathrm{M})$ for $24 \mathrm{~h}$. Cells were stained with DAPI (50 ng/ml), and visualized under a fluorescence microscope. The slides were viewed at x1000 magnification on an Olympus fluorescence microscope (BX40 with MagnaFire CCD camera).

Statistical analysis. The mean and SD were calculated for each experimental group. Differences between groups were analyzed by one or two-way ANOVA using PRISM statistical analysis software (GrafPad Software, Inc., San Diego, CA). Significant differences among groups were calculated at $\mathrm{P}<0.05$.

\section{Results}

Inhibitors of AKT and ERK synergistically/additively inhibit migration and capillary tube formation of HUVEC cells, and further enhance the inhibitory effects of sulforaphane on migration and capillary tube formation. We first measured the effect of SFN treatment on invasion potential (migration) of HUVEC cells using a modified Boyden Chamber assay (Fig. 1A). In DMSO-treated controls, a large fraction of HUVEC migrated to the bottom face of the membrane. Treatment of chambers with AKT inhibitor, ERK inhibitor (MEK1/2 inhibitor PD98059) or sulforaphane resulted in inhibition of migration of HUVECs. The combination of AKT inhibitor and PD98059 synergistically/additively inhibited 
A
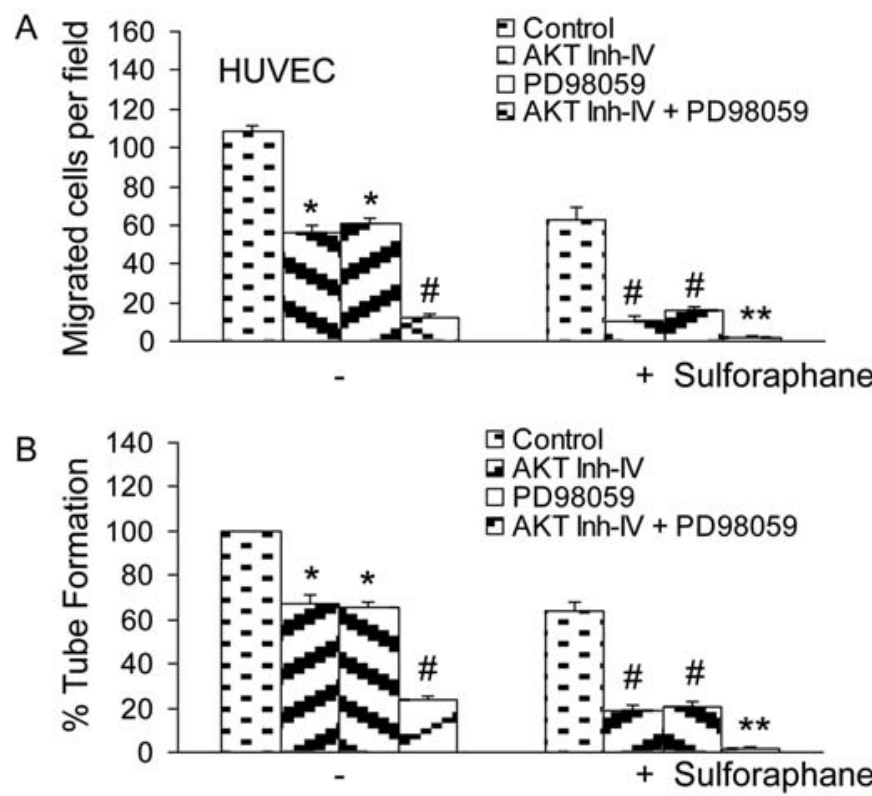

Figure 1. Sulforaphane inhibits migration and capillary tube formation by HUVEC cells. (A) Migration of HUVEC cells was assessed using Transwell Boyden chamber containing a polycarbonated filter. HUVECs $\left(4 \times 10^{4}\right.$ cells $)$ were pretreated with AKT inhibitor IV $(1 \mu \mathrm{M})$ and/or MEK1/2 inhibitor PD98059 $(10 \mu \mathrm{M})$ for $2 \mathrm{~h}$, followed by treatment with sulforaphane $(20 \mu \mathrm{M})$ or DMSO (control). Migration through the membrane was determined after $24 \mathrm{~h}$ of incubation at $37^{\circ} \mathrm{C}$. Cells that had migrated to the lower chamber were fixed with $90 \%$ ethanol, stained with hematoxylin and eosin quantified by counting the number of cells under a microscope. Data represent mean $\pm \mathrm{SD}$. * ${ }^{* *}$, or \#, significantly different from control, $\mathrm{P}<0.05$. (B) HUVECs $\left(10 \times 10^{4}\right)$ were seeded in 24 -well plates containing matrigel, and pretreated with AKT inhibitor IV and/or MEK1/2 inhibitor PD98059 for $2 \mathrm{~h}$, followed by treatment with sulforaphane $(20 \mu \mathrm{M})$ or DMSO (control) for $24 \mathrm{~h}$. Capillary tubes were counted under a microscope. Data represent mean $\pm \mathrm{SD}$. *, **, or \#, significantly different from control, $\mathrm{P}<0.05$.

cell migration. Interestingly, the inhibitory effects of SFN on cell migration were further enhanced in the presence of AKT inhibitor and/or PD98059.

We next examined the effects of PI3K/AKT and MEK/ ERK pathway on capillary tube formation by HUVEC on growth factor-reduced matrigel. The data revealed that AKT inhibitor, PD98059 and SFN alone inhibited capillary tube formation (Fig. 1B). The combination of AKT inhibitor and PD98059 synergistically inhibited capillary tube formation. Interestingly, the inhibitory effects of SFN on capillary tube formation were further enhanced in the presence of AKT inhibitor and/or PD98059. These results indicated that PI3K/ AKT and MEK/ERK pathways act synergistically/additively to inhibit migration and capillary tube formation by HUVEC cells.

FOXO1-TM and FOXO3A-TM (phosphorylation deficient mutants of FOXO) enhance the inhibitory effects of sulforaphane on migration and capillary tube formation by HUVEC cells. We next examined the involvement of FOXO transcription factors in SFN-induced migration and capillary tube formation by HUVEC cells (Fig. 2). Overexpression of phosphorylation deficient mutants of FOXO (FOXO1-TM and FOXO3a-TM) inhibited migration and capillary tube
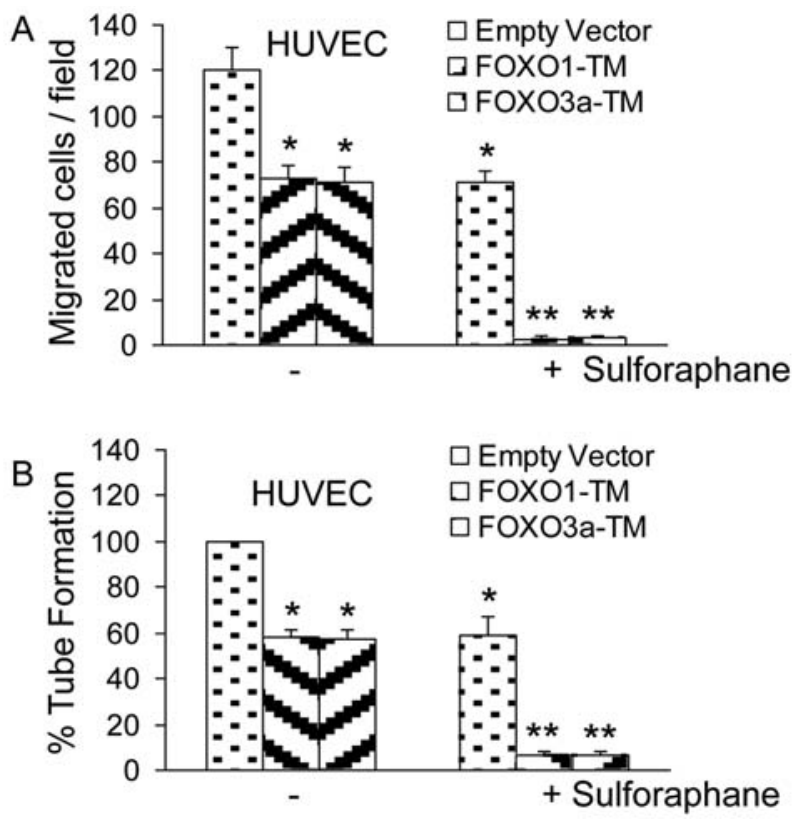

Figure 2. Phosphorylation deficient mutants of FOXO enhance the inhibitory effects of sulforaphane on migration and capillary tube formation. (A) HUVEC $\left(4 \times 10^{4}\right)$ cells were transiently transfected with empty vector, FOXO1-TM or FOXO3A-TM along with pCMV-LacZ vector (as transfection control) and treated with or without sulforaphane $(20 \mu \mathrm{M})$. Migration through the membrane was determined after $24 \mathrm{~h}$ of incubation at $37^{\circ} \mathrm{C}$. Cells that had migrated to the lower chamber were fixed with $90 \%$ ethanol, stained with hematoxylin and eosin, quantified by counting the number of cells under a microscope. Data represent mean $\pm \mathrm{SD}$. *, **, significantly different from control, $\mathrm{P}<0.05$. (B) HUVEC $\left(4 \times 10^{4}\right)$ cells were transiently transfected with empty vector, FOXO1-TM or FOXO3A-TM along with pCMV-LacZ vector (as transfection control) and treated with or without sulforaphane $(20 \mu \mathrm{M})$ for $24 \mathrm{~h}$. Capillary tubes were counted under a microscope. Data represent mean \pm SD. * , **, significantly different from control, $\mathrm{P}<0.05$.

formation by HUVEC cells. Furthermore, overexpression of FOXO1-TM and FOXO3a-TM further enhanced the inhibitory effects of SFN on migration and capillary tube formation. These data suggest that FOXO transcription factors may play major role in angiogenesis.

Inhibitors of AKT and ERK synergistically/additively induce FOXO transcriptional activity, and further enhanced FOXO transcriptional activity induced by sulforaphane. We next examined whether inhibition of PI3K/AKT and MEK/ERK pathways synergistically act to induce FOXO transcriptional activity, and inhibition of these two pathways further enhance SFN-induced FOXO activity (Fig. 3). AKT inhibitor, MEK1/2 inhibitor PD98059 and SFN alone induced FOXO transcriptional activity. The combination of AKT inhibitor and PD98059 synergistically induced FOXO activity. Furthermore, the combination of AKT inhibitor and/or PD98059 with SFN further enhanced FOXO transcriptional activity. Inhibitors of AKT and MEK synergistically enhanced nuclear translocation of FOXO3a in HUVECs (data not shown). These data suggest that inhibition of PI3K/AKT and MEK1/2/ERK pathways act synergistically to induce FOXO transcriptional activity, and inhibition of these two pathways further enhance SFN-induced FOXO activity. 


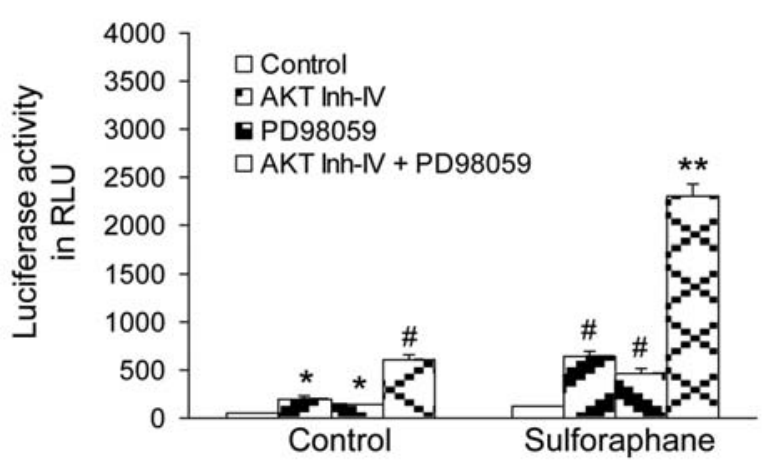

Figure 3. Inhibition of PI3-K/AKT and MEK/ERK pathways synergistically/ additively enhanced sulforaphane-induced FOXO activity in HUVEC cells. HUVEC $\left(4 \times 10^{4}\right)$ cells were transiently transfected with 6 X DBE-luciferase construct for $24 \mathrm{~h}$ (33). After transfection, HUVEC cells were pretreated with AKT inhibitor IV $(1 \mu \mathrm{M})$ and/or MEK1/2 inhibitor PD98059 $(10 \mu \mathrm{M})$ for $2 \mathrm{~h}$, followed by treatment with or without sulforaphane $(20 \mu \mathrm{M})$ for $24 \mathrm{~h}$. Cells were harvested for firefly/Renilla luciferase assays using the Dual-Luciferase Reporter Assay System (Promega). Luciferase counts were normalized using Renilla luciferase transfection control (pRL-TK; Promega). Data represent the mean \pm S.D. *,\#,**, significantly different from respective controls, $\mathrm{P}<0.05$.

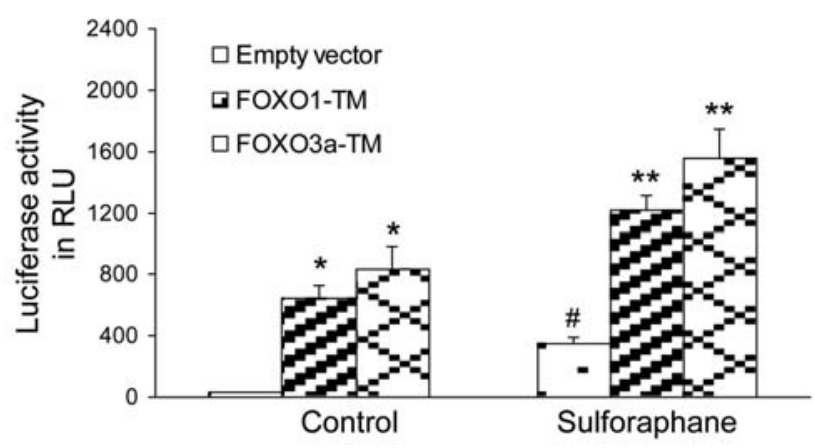

Figure 4. Phosphorylation deficient mutants of FOXO enhance sulforaphaneinduced FOXO transcriptional activity in HUVEC cells. HUVEC cells were transiently transfected with empty vector or constructs encoding FOXO1-TM, or FOXO3a-TM together with 6X DBE-luciferase for $24 \mathrm{~h}$ (33). Luciferase counts were normalized using Renilla luciferase transfection control (pRL-TK; Promega). After transfection, cells were washed with RPMI, treated with sulforaphane $(20 \mu \mathrm{M})$ for $24 \mathrm{~h}$, and harvested for firefly/Renilla luciferase assays using the Dual-Luciferase Reporter Assay System (Promega). Data represent the mean \pm S.D. *, \#, **, significantly different from respective controls, $\mathrm{P}<0.05$.

Phosphorylation deficient mutants of FOXO induce FOXO transcriptional activity, and further enhanced sulforaphaneinduced FOXO activity in HUVEC cells. We next examined whether SFN induces transcriptional activation of FOXO in the presence or absence FOXO1-TM, or FOXO3a-TM (phosphorylation deficient triple mutant) (Fig. 4). HUVEC cells were transfected with wild-type FOXO promoter linked to a luciferase reporter gene in the presence or absence of plasmids expressing FOXO1-TM, or FOXO3a-TM. After transfection, cells were treated with SFN for $24 \mathrm{~h}$, and luciferase activity was measured. Transfection of cells with plasmids expressing FOXO1-TM, or FOXO3a-TM induced FOXO transcriptional activity compared with the empty vector (control). SFN-induced FOXO transcriptional activity was further enhanced in the presence of phosphorylation deficient mutants of FOXO1, or FOXO3a. These data indicate that FOXO transcription factors can mediate the antiangiogenic effects of SFN.

\section{Discussion}

The consumption of cruciferous vegetables has long been associated with a reduced risk in the occurrence of cancer at various sites, including the prostate, lung, breast and colon (3). This protective effect is attributed to isothiocyanates present in these vegetables (3). Recent studies have demonstrated that sulforaphane (SFN) present in broccoli inhibit tumor growth and angiogenesis $(6,9,34,35)$. The major mechanism by which SFN protects cells was traditionally thought to be through Nrf2-mediated induction of phase 2 detoxification enzymes that elevate cell defense against oxidative damage and promote the removal of carcinogens. However, it is becoming clear that there are multiple mechanisms activated in response to SFN, including suppression of cytochrome P450 enzymes, induction of apoptotic pathways, suppression of cell cycle progression, inhibition of angiogenesis and anti-inflammatory activity. Moreover, these mechanisms seem to have some degree of interaction to synergistically afford chemoprevention.

Sulforaphane has been shown to suppress diverse cellular events associated with each step of carcinogenesis, i.e., tumor initiation, promotion and progression (3). We have recently demonstrated that SFN inhibited tumor growth and markers of angiogenesis and metastasis in an orthotopic model of prostate cancer (9). Although FOXO transcription factors have recently been shown to inhibit angiogenesis, their involvement in antiangiogenic effects of SFN has never been examined. To the best of our knowledge, this is the first study to demonstrate that inhibition of PI3K/AKT and MEK/ ERK pathways acted synergistically to induce activation of FOXO transcription factors and enhanced the antiangiogenic effects of SFN in HUVEC cells. Inhibitors of AKT and MEK synergistically enhanced nuclear translocation of FOXO3a in HUVECs. Specifically, cell migration and capillary tube formation was inhibited by inhibitors of AKT and MEK, and phsophorylation deficient mutants of FOXO. Inhibitors of AKT and MEK further enhanced antiangiogenic effects of SFN.

Activation of PI3K/AKT and MEK/ERK pathways may enhance angiogenesis, and inhibition of these two pathways may inhibit angiogenesis through regulation of FOXO transcription factors. In the present study, MEK inhibitor, AKT inhibitor, and phosphorylation deficient mutants of FOXO activated FOXO transcription factor resulting in inhibition of capillary tube formation and migration by HUVECs. SFN further enhanced the inhibitory effects of MEK inhibitor, and AKT inhibitor on capillary tube formation and cell migration by HUVECs. These data suggest that anti-angiogenic effects of SFN can be mediated through simultaneous inhibition of MEK/ERK and PI3K/AKT pathways, and these two pathways can additively or synergistically converge to regulate activation FOXO transcription factor. The activated FOXO transcription factors will inhibit angiogenesis and thus tumor growth. Similar to the present study, we have recently demon- 
strated that another chemopreventive agent EGCG can modulate the expression of genes known to play a role in the cancer progression, invasion, metastasis and angiogenesis $(31,36)$. We have also demonstrated that inhibition of PI3K/AKT and MEK/ERK pathways converge to inhibit angiogenesis through activation of FOXO transcription factors, and anti-angiogenic effects of EGCG were regulated by FOXO (30).

Angiogenesis is a critical step in tumor growth and metastasis $(37,38)$, which are regulated closely by the local increase of the activity of a variety of angiogenic factors. During angiogenesis, endothelial cells migrate, proliferate, organize into tube-like structures, and play an active role in tissue remodeling. Substantial evidence also suggests that stroma cells adjacent to the cancer cells, including fibroblasts and inflammatory cells such as macrophages, neutrophils, and lymphocytes, can interact with cancer cells and express angiogenic factors $(39,40)$. We have recently demonstrated that SFN can inhibit the activation of NFKB and its gene products such as VEGF, HIF-1 $\alpha$, MT1-MMP, MMP-2, MMP-9, COX-2, IL-6 and IL-8 in an orthotopic model of prostate cancer (9). These tumor derived factors can affect angiogenesis and metastasis. In the present study, SFN inhibited cell migration and capillary tube formation which are important events for angiogenesis.

FOXO transcription factors suppress tumor growth because of their effects on cell-cycle, apoptosis, angiogenesis, DNA-damage and reactive oxygen species. FOXO proteins have also been implicated in the negative regulation of signaling by the hypoxia-inducible factor 1 (HIF-1) during vascular development, raising the possibility that the FOXO proteins suppress not only tumor formation but also tumor angiogenesis. FOXO1 and FOXO3a are the most abundant FOXO isoforms in mature endothelial cells and that overexpression of constitutively active Foxo1 or Foxo3a significantly inhibits endothelial cell migration and tube formation in vitro (41). Silencing of either FOXO1 or FOXO3a gene expression led to a profound increase in the migratory and sprout-forming capacity of endothelial cells. The FOXO1 deficient mice died around embryonic day 11 because of defects in the branchial arches and impaired vascular development of embryos and yolk sacs (32). Gene expression profiling showed that FOXO1 and FOXO3a specifically regulate a non-redundant but overlapping set of angiogenesis- and vascular remodeling-related genes. FOXO1 regulates angiopoietin-2 (Ang-2), whereas eNOS, which is essential for postnatal neovascularization, is regulated by FOXO1 and FOXO3a. Consistent with these findings, constitutively active FOXO1 and FOXO3a repressed eNOS protein expression. In vivo, Foxo3a deficiency increased eNOS expression and enhanced postnatal vessel formation and maturation. Thus, our data suggest an important role for FOXO transcription factors in the regulation of angiogenesis.

In conclusion, we have demonstrated that inhibition of PI3K/AKT and MEK/ERK pathways interact synergistically to activate FOXO transcription factors which, in turn, inhibit angiogenesis. Furthermore, inhibition of both of these pathways further enhances the anti-angiogenic effects of sulforaphane. The activation of FOXO transcription factors through inhibition of MEK/ERK and PI3K/AKT pathways may have physiological significance for tumor growth through regulation of angiogenesis. Our data strongly support the role of SFN as an antiangiogenic agent.

\section{Acknowledgements}

We thank our lab members for critical reading of the manuscript. We also thank Dr Noboru Motoyama (National Institute for Longevity Sciences, Obu, Aichi, Japan) and Dr Tatsuo Furuyama (Sonoda Women's University, Amagasaki, Hyogo, Japan) for providing FOXO expression plasmids and FOXO-luciferase construct (pGL3-6X DBE), respectively.

\section{References}

1. Clarke JD, Dashwood RH and Ho E: Multi-targeted prevention of cancer by sulforaphane. Cancer Lett 269: 291-304, 2008.

2. Nian H, Delage B, Ho E and Dashwood RH: Modulation of histone deacetylase activity by dietary isothiocyanates and allyl sulfides: studies with sulforaphane and garlic organosulfur compounds. Environ Mol Mutagen 50: 213-221, 2009.

3. Kelloff GJ, Crowell JA and Steele VE: Progress in cancer chemoprevention: development of diet-derived chemopreventive agents. J Nutr 130: 467S-471S, 2000.

4. Singh SV, Warin R and Xiao D: Sulforaphane inhibits prostate carcinogenesis and pulmonary metastasis in TRAMP mice in association with increased cytotoxicity of natural killer cells. Cancer Res 69: 2117-2125, 2009

5. Gills JJ, Jeffery EH, Matusheski NV, Moon RC, Lantvit DD and Pezzuto JM: Sulforaphane prevents mouse skin tumorigenesis during the stage of promotion. Cancer Lett 236: 72-79, 2006.

6. Kallifatidis G, Rausch V and Baumann B: Sulforaphane targets pancreatic tumor-initiating cells by NF-\{kappa\}B-induced antiapoptotic signaling. Gut 4: 123-136, 2008.

7. Myzak MC, Dashwood WM, Orner GA, Ho E and Dashwood RH: Sulforaphane inhibits histone deacetylase in vivo and suppresses tumorigenesis in Apc-minus mice. FASEB J 20: 506-508, 2006.

8. Pham NA, Jacobberger JW, Schimmer AD, Cao P, Gronda M and Hedley DW: The dietary isothiocyanate sulforaphane targets pathways of apoptosis, cell cycle arrest, and oxidative stress in human pancreatic cancer cells and inhibits tumor growth in severe combined immunodeficient mice. Mol Cancer Ther 3: 1239-1248, 2004.

9. Shankar S, Ganapathy S and Srivastava RK: Sulforaphane enhances the therapeutic potential of TRAIL in prostate cancer orthotopic model through regulation of apoptosis, metastasis and angiogenesis. Clin Cancer Res 14: 1-16, 2008.

10. Zhang Y, Kensler TW, Cho CG, Posner GH and Talalay P: Anticarcinogenic activities of sulforaphane and structurally related synthetic norbornyl isothiocyanates. Proc Natl Acad Sci USA 91: 3147-3150, 1994.

11. Fahey JW, Zhang Y and Talalay P: Broccoli sprouts: an exceptionally rich source of inducers of enzymes that protect against chemical carcinogens. Proc Natl Acad Sci USA 94: 10367-10372, 1997.

12. Fahey JW, Haristoy X and Dolan PM: Sulforaphane inhibits extracellular, intracellular, and antibiotic-resistant strains of Helicobacter pylori and prevents benzo[a]pyrene-induced stomach tumors. Proc Natl Acad Sci USA 99: 7610-7615, 2002 .

13. Chung FL, Conaway CC, Rao CV and Reddy BS: Chemoprevention of colonic aberrant crypt foci in Fischer rats by sulforaphane and phenethyl isothiocyanate. Carcinogenesis 21: 2287-2291, 2000.

14. Kim SJ, Kang SY, Shin HH and Choi HS: Sulforaphane inhibits osteoclastogenesis by inhibiting nuclear factor-kappaB. Mol Cells 20: 364-370, 2005.

15. Hanlon N, Coldham N and Gielbert A: Absolute bioavailability and dose-dependent pharmacokinetic behaviour of dietary doses of the chemopreventive isothiocyanate sulforaphane in rat. Br J Nutr 99: 559-564, 2008. 
16. Rushmore TH and Kong AN: Pharmacogenomics, regulation and signaling pathways of phase I and II drug metabolizing enzymes. Curr Drug Metab 3: 481-490, 2002.

17. Misiewicz I, Skupinska K, Kowalska E, Lubinski J and Kasprzycka-Guttman T: Sulforaphane-mediated induction of a phase 2 detoxifying enzyme $\mathrm{NAD}(\mathrm{P}) \mathrm{H}$ :quinone reductase and apoptosis in human lymphoblastoid cells. Acta Biochim Pol 51: 711-721, 2004.

18. Anderson MJ, Viars CS, Czekay S, Cavenee WK and Arden KC: Cloning and characterization of three human forkhead genes that comprise an FKHR-like gene subfamily. Genomics 47: 187-199, 1998

19. Van Der Heide LP, Hoekman MF and Smidt MP: The ins and outs of FoxO shuttling: mechanisms of FoxO translocation and transcriptional regulation. Biochem J 380: 297-309, 2004.

20. Nakamura N, Ramaswamy S, Vazquez F, Signoretti S, Loda $\mathrm{M}$ and Sellers WR: Forkhead transcription factors are critical effectors of cell death and cell cycle arrest downstream of PTEN. Mol Cell Biol 20: 8969-8982, 2000.

21. Cappellini A, Tabellini G and Zweyer M: The phosphoinositide 3-kinase/Akt pathway regulates cell cycle progression of HL60 human leukemia cells through cytoplasmic relocalization of the cyclin-dependent kinase inhibitor p27(Kip1) and control of cyclin D1 expression. Leukemia 17: 2157-2167, 2003.

22. Dijkers PF, Medema RH, Lammers JW, Koenderman L and Coffer PJ: Expression of the pro-apoptotic Bcl-2 family member Bim is regulated by the forkhead transcription factor FKHR-L1. Curr Biol 10: 1201-1204, 2000

23. Gilley J, Coffer PJ and Ham J: FOXO transcription factors directly activate bim gene expression and promote apoptosis in sympathetic neurons. J Cell Biol 162: 613-622, 2003.

24. Brunet A, Bonni A and Zigmond MJ: Akt promotes cell survival by phosphorylating and inhibiting a Forkhead transcription factor. Cell 96: 857-868, 1999

25. Tang TT, Dowbenko D and Jackson A: The forkhead transcription factor AFX activates apoptosis by induction of the BCL-6 transcriptional repressor. J Biol Chem 277: 14255-14265, 2002.

26. Chaudhuri D, Orsulic S and Ashok BT: Antiproliferative activity of sulforaphane in Akt-overexpressing ovarian cancer cells. Mol Cancer Ther 6: 334-345, 2007.

27. Hu R, Khor TO and Shen G: Cancer chemoprevention of intestinal polyposis in ApcMin/ ${ }^{+}$mice by sulforaphane, a natural product derived from cruciferous vegetable. Carcinogenesis: 2038-2046, 2006
28. Asada S, Daitoku H and Matsuzaki H: Mitogen-activated protein kinases, Erk and p38, phosphorylate and regulate Foxo1. Cell Signal 19: 519-527, 2007.

29. Yang LQ, Fang DC, Wang RQ and Yang SM: Effect of NFkappaB, survivin, Bcl-2 and Caspase 3 on apoptosis of gastric cancer cells induced by tumor necrosis factor related apoptosis inducing ligand. World J Gastroenterol 10: 22-25, 2004.

30. Shankar S, Chen Q and Srivastava RK: Inhibition of PI3K/AKT and MEK/ERK pathways act synergistically to enhance antiangiogenic effects of EGCG through activation of FOXO transcription factor. J Mol Signal 3: 7-16, 2008.

31. Shankar S, Ganapathy S, Hingorani SR and Srivastava RK: EGCG inhibits growth, invasion, angiogenesis and metastasis of pancreatic cancer. Front Biosci 13: 440-452, 2008.

32. Furuyama T, Kitayama K and Shimoda Y: Abnormal angiogenesis in Foxo1 (Fkhr)-deficient mice. J Biol Chem 279: 34741-34749, 2004.

33. Furukawa-Hibi Y, Kobayashi Y, Chen C and Motoyama N: FOXO transcription factors in cell-cycle regulation and the response to oxidative stress. Antioxid Redox Signal 7: 752-760, 2005.

34. Asakage M, Tsuno NH and Kitayama J: Sulforaphane induces inhibition of human umbilical vein endothelial cells proliferation by apoptosis. Angiogenesis 9: 83-91, 2006.

35. Jackson SJ, Singletary KW and Venema RC: Sulforaphane suppresses angiogenesis and disrupts endothelial mitotic progression and microtubule polymerization. Vascul Pharmacol 46: 77-84, 2007.

36. Shankar S, Suthakar G and Srivastava RK: Epigallocatechin3 -gallate inhibits cell cycle and induces apoptosis in pancreatic cancer. Front Biosci 12: 5039-5051, 2007.

37. Folkman J: Fundamental concepts of the angiogenic process. Curr Mol Med 3: 643-651, 2003

38. Folkman J: Angiogenesis and proteins of the hemostatic system. J Thromb Haemost 1: 1681-1684, 2003.

39. Fukumura D, Xavier R and Sugiura T: Tumor induction of VEGF promoter activity in stromal cells. Cell 94: 715-725, 1998

40. Connolly DT, Stoddard BL, Harakas NK and Feder J: Human fibroblast-derived growth factor is a mitogen and chemoattractant for endothelial cells. Biochem Biophys Res Commun 144: 705-712, 1987.

41. Potente M, Urbich C and Sasaki K: Involvement of Foxo transcription factors in angiogenesis and postnatal neovascularization. J Clin Invest 115: 2382-2392, 2005. 